\title{
High Speed Constant Force Milling Based on Fuzzy Controller and BP Neural Network
}

\author{
Yunlin Huang ${ }^{1}$ and Juntang Yuan ${ }^{1}$ \\ ${ }^{1}$ School of Mechanical Engineering, Nanjing University of Science and Technology \\ Nanjing 210094, China
}

mashuangyunlin@163.com,mc106@mail.njust.edu.cn

\begin{abstract}
In order to meet the high cutting rate and high surface quality requirement, put forward the fuzzy control, on-line adjusting the cutting feed rate method to realize a constant force in CNC high speed milling. The input variables of a fuzzy controller are the difference between the reference forces and the actual cutting forces and the change rate of the deviation, after fuzzification, fuzzy inference and defuzzification, the output variable is the change of per tooth feed. The control strategy of on-line adjusting fuzzy rules and on-line self-adaptiving the output scaling factor is used in a fuzzy controller. The CNC high speed milling process model is established by 3-3-5-1 type BP neural network, and the input variables are cutting parameters, and the output variable is the actual cutting forces. Combining the fuzzy controller and the BP neural network, high speed milling constant force control model is constructed. By simulation and practical verification, metal removal rate is increased significantly, and the surface quality is improved substantially.
\end{abstract}

Keywords: Fuzzy control, BP neural network, High speed milling, constant forces control

\section{Introduction}

The use of computer numerical control (CNC) machining centers has expanded rapidly through the years. A great advantage of the $\mathrm{CNC}$ machining center is that it reduces the skill requirements of machine operators. However, a common drawback of CNC end milling is that its operating parameter such as spindle speed or feedrate is prescribed conservatively either by a part programmer or by a relatively static database in order to preserve the tool.

As a result, many CNC systems run under inefficient operating conditions. For this reason, a CNC machine tool control system, which provides on-line adjustment of the operating parameters, is being studied with interest. These systems can be classified into three types: a geometric adaptive compensation (GAC) system; an adaptive control optimization (ACO) system; and an adaptive control constraints (ACC) system. GAC systems enhance part precision by applying real time geometric error compensation for imprecision caused by varying machine temperature, imprecise machine geometry, tool wear and other factors [1]. However, due to the difficulty in on-line measurement of tool wear and machine tool temperature, there are no commercial GAC systems available [2]. ACO systems and ACC systems enhance productivity by applying an adaptive control technique to vary then machining variables in real time [3]. ACO systems set up the most effective cutting condition for the present cutting environment. For this purpose, ACO systems require on-line measurement of tool wear. Unfortunately, adaptive control alone cannot effectively control cutting forces. There is no controller that can respond quickly enough to sudden changes in the cut geometry to eliminate large spikes in cutting forces. Therefore, we implement on-line 
adaptive control in conjunction with off-line optimization.

In the process of CNC high speed milling, the most effective way to improve cutting efficiency is to improve metal removal rate (MRR) within the scope of the machine tool, cutting tool and workpiece system. Among the many ways to improve the metal removal rate, online adjusting the feed rate is the main way; aims to achieve constant forces control [4-6]. But in the actual process of high speed milling, due to the change of cutting parameters, the influence of the dynamic characteristics of machine tool and other factors, makes the cutting process is highly nonlinear, time-varying and uncertainty. The traditional CNC modeling method is difficult to obtain the ideal effect.

This paper studies the constant force control process in high-speed milling using the fuzzy control method and BP neural network. Compared with traditional control methods, a fuzzy control system doesn't need to make precise mathematical model, only establishing the control model directly according to the rules of experience. According to the deviation and the deviation change rate of the measured value and the set value, the optimal control of the output value is gotten. The result has strong robustness, especially adapting to the uncertain and nonlinear system. The high speed milling model is established using BP neural network model based on multiple sets of cutting force measured under different high speed cutting parameters. Constant force cutting can give full play to the potential process of CNC milling machine and machining efficiency, reducing the tool wear and improving the machining accuracy and surface quality. The basic idea in this paper is to make the material removal rate as high as possible and maintain cutting force as close as possible to the given reference force through online adjusting per tooth feed. The correctness of the model is verified by simulation and experiment.

\section{Design of the Fuzzy Control Algorithm}

The basic idea of fuzzy control is to use the machine to simulate the control system, summarize some rules through the expert experience and to design the controller according to these rules. Because the person's experience is generally described by natural language, thus the rules based on the experience are fuzzy. However, the fuzzy rules of the language can be converted to the numerical computing using the fuzzy theory, fuzzy language variable and fuzzy logic reasoning knowledge, which can use the computer to complete the specific implementation of these rules, achieving some objects by machine instead of human control.

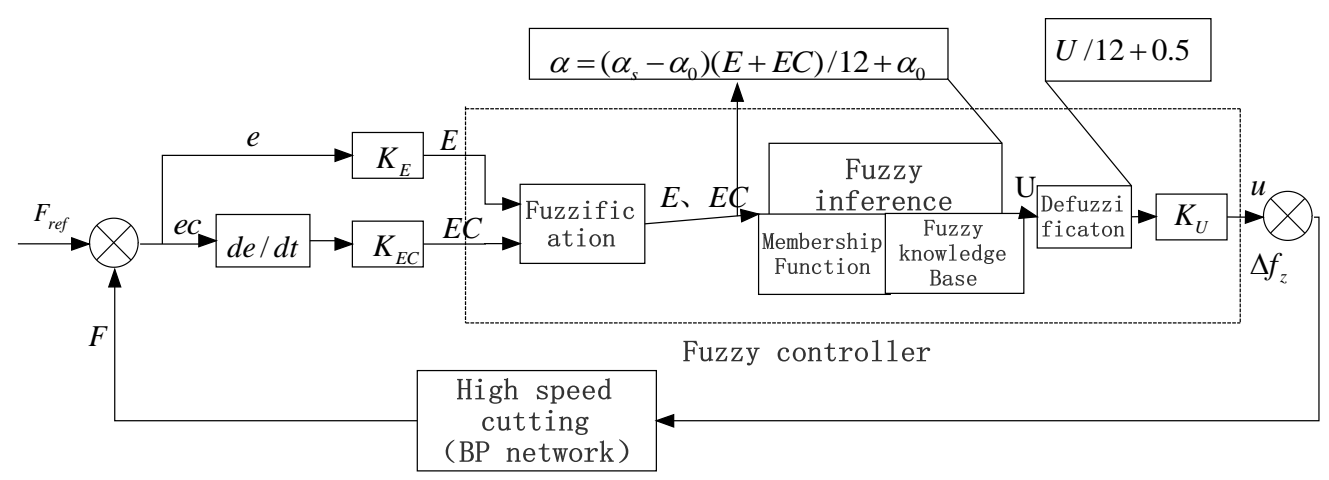

Figure 1. High speed constant force milling control schematic diagram based on fuzzy control and BP neural network 
Figure 1 is a typical two-input and single-output schematic diagram based on the fuzzy control in high speed milling. Controller's input linguistic variables are the difference between reference forces $F_{\text {ref }}$ and actual cutting forces $F$ and the change rate of the deviation, and the output linguistic variable is the per tooth feed variation $\Delta f_{z}$. The process of fuzzy control is to find the fuzzy relationship between the per tooth feed variation $\Delta f_{z}$ and deviation e, deviation change rate ec, detecting e and ec continuously, on-line modifying $\Delta f_{z}$ according to the fuzzy control rules, to meet different e and ec to the different requirement of the controller parameters, so that the controlled object has a good dynamic and static performance.

In Figure1, e is a deviation between an expectable value and the actual value, ec is the change rate of deviation, E, EC are fuzzy controller input values, $K_{E} 、 K_{E C}$ are quantitative factors, $K_{U}$ is the proportional output factor.

$$
\begin{aligned}
& e(i)=F(i)-F_{r e f}(i) \\
& e c(i)=[e(i)-e(i-1)] / d t
\end{aligned}
$$

\subsection{Fuzzification}

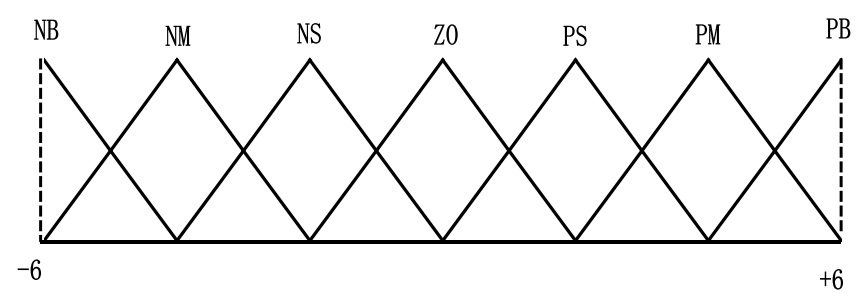

Figure 2. Subordinating degree function of input and output variables

By the method of triangle membership function, the input and output variables have been fuzzily transformed as shown in Figure 2. Variables are subordinated the following fuzzy subset: negative big (NB), negative medium (NM), negative small (NS), zero (ZO), positive small (PS), positive medium (PM), positive big (PB). The discrete domain of E, EC, $\Delta f_{z}$ is $[-6,-5,-4,-3,-2,-1,0,+1,+2,+3,+4,+5,+6]$.

\subsection{Fuzzy inference}

The function of fuzzy inference is to inference the input variables according to the fuzzy knowledge base composed of a series of linguistic rules, and obtain the optimal values. The conventional fuzzy control rules do not have adaptability, easily leading to zero limit cycle oscillation phenomenon. Unchanged the fuzzy control rules are difficult to satisfy the requirement of the nonlinear and time-varying dynamic system. So the performance of a self-tuning fuzzy controller is required.

Deviation E and deviation change rate EC in different stage has different effects on the controller. Control rules can be described by the following formula:

$$
U=<\alpha E+(1-\alpha) E C>\quad \alpha \in[0,1]
$$


In formula (3), $\alpha$ is a correction factor, used to adjust the E and EC in different stages.

$$
\alpha=\left(\alpha_{s}-\alpha_{0}\right)(E+E C) /(2 N)+\alpha_{0}
$$

In formula (4), $\mathrm{N}=6,0 \leq(\mathrm{E}+\mathrm{EC}) / 12 \leq 1, \alpha_{s}=0.7, \alpha_{0}=0.5$.

\subsection{Defuzzification}

The control output of fuzzy inference is a fuzzy membership function, and it reflects the fuzziness of the control language, which is a different combination of values. In practice to control an object must be in the moment engraved with a certain amount of control, to find one of the most outputs from the fuzzy membership functions to represent the fuzzy set of precise quantity, namely defuzzification judgment.

Defuzzification is done by using the weighted average method. Each element $x_{i}(i=1,2 \ldots, n)$ in a domain serves as the weighted coefficient of output of membership $\mu_{\breve{U}_{1}}\left(x_{i}\right)$ in the fuzzy $\operatorname{set} \breve{U}_{1}$, namely

$$
x_{0}=\frac{\sum_{i=1}^{n} x_{i} \mu_{\breve{U}_{1}}\left(x_{i}\right)}{\sum_{i=1}^{n} \mu_{\breve{U}_{1}}\left(x_{i}\right)}
$$

The corresponding accurate quantity $x_{0}$ can be looked up by the $\mathrm{U}$ assignment table. This value is $\Delta f_{z}$.In defuzzification, the selection of the output scaling factor $K_{U}$ is very important. Procky[11] points out that the stability and response speed when changing the values of $K_{E}$ and $K_{E C}$ are contradictory to each other, while the adjustment of an output scaling factor $K_{U}$ is relatively simple, and it can be applied directly to the output control amount.

In high-speed milling constant force control tests,

$$
K_{U}=\frac{F_{r e f}}{c_{s} k_{p}}
$$

In formula (6), $c_{s}=0.5$ and $k_{p}$ is a gain value of the controlled object, $k_{p}$ is estimated by using integral recursive method.

$$
\left\{\begin{array}{c}
E_{r}(i)=F(i)-k_{p}(i-1) u(i-1) \\
k_{p}(i)=k_{p}(i-1)+c E_{r}(i)
\end{array}\right.
$$

In formula (7), $E_{r}$ is the estimated deviation of the cutting force, $F(i)$ is milling force in the $i$ moment, $c$ is a small constant, $c=0.04$, and $u(i)$ is the feed speed, $u(i)=K_{U}(i)[U(i) / 12+0.5]$.

The adaptive fuzzy controller is constructed step by step. High speed milling constant force control can be realized when completed. 


\section{High-speed Milling Process Modeling Based on Neural Network}

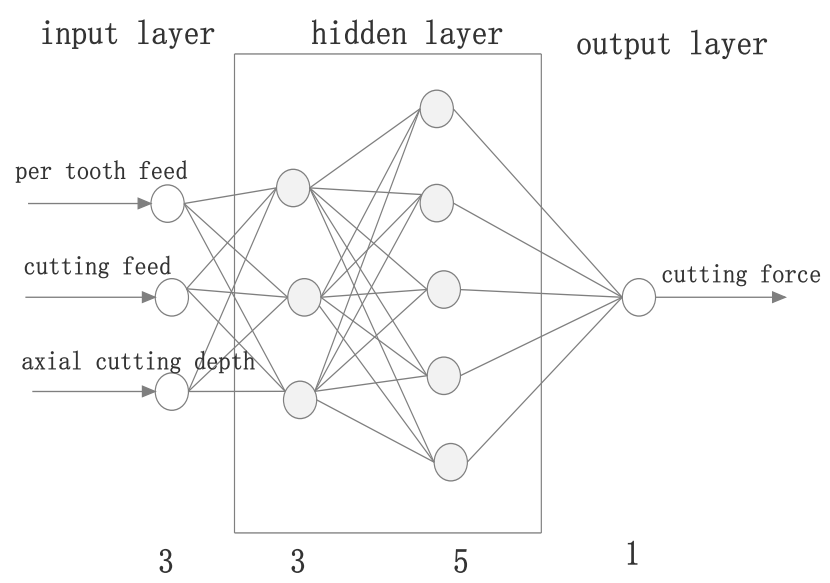

Figure 3. The model of BP neural network in high speed milling process

Due to the nonlinear and time-varying characteristics in a high speed milling process, it's difficult to use a mathematical model to describe accurately; therefore the BP neural network is used to modeling the process of high speed milling, to establish the nonlinear relationship between processing parameters and the cutting force. The mathematical model is shown in Figure 3. Neural network uses the S-type linear function, and hidden layer uses the Sigmoid transfer function, and the output layer is purelin linear transfer function. There are many types of a neural network training algorithm, through the comprehensive contrast each training algorithm convergence speed, memory consumption and the suitable network, network training function is selected as Levenberg -Marquardt function, namely trainlm function. Number of input neurons is 3 , represent the processing parameters (per tooth feed $f_{z}$, cutting speed $v$, axial cutting depth $a_{p}$ ); Number of the output neuron is 1 , represents the milling force. Node number of hidden layer neurons has a greater influence on the performance of neural network, too much of the node number will cause the training time and testing error increases, and too little will cause a decline in network fault tolerance, the correct recognition rate and the generalization ability. In order to achieve faster convergence speed and obtain high testing precision, after many tests, the number of hidden layer is 2 , the neuron number is 3 and 5, respectively. The milling force values of different cutting parameters recorded in experiment, choosing 500 groups of data for neural network training, until satisfactory results are obtained. The neural network can be encapsulated using the gensim command in Matlab, which can be used in the simulation in Matlab/Simulink. 


\section{Fuzzy Control Constant Force Simulation Based on Matlab/Simulink}

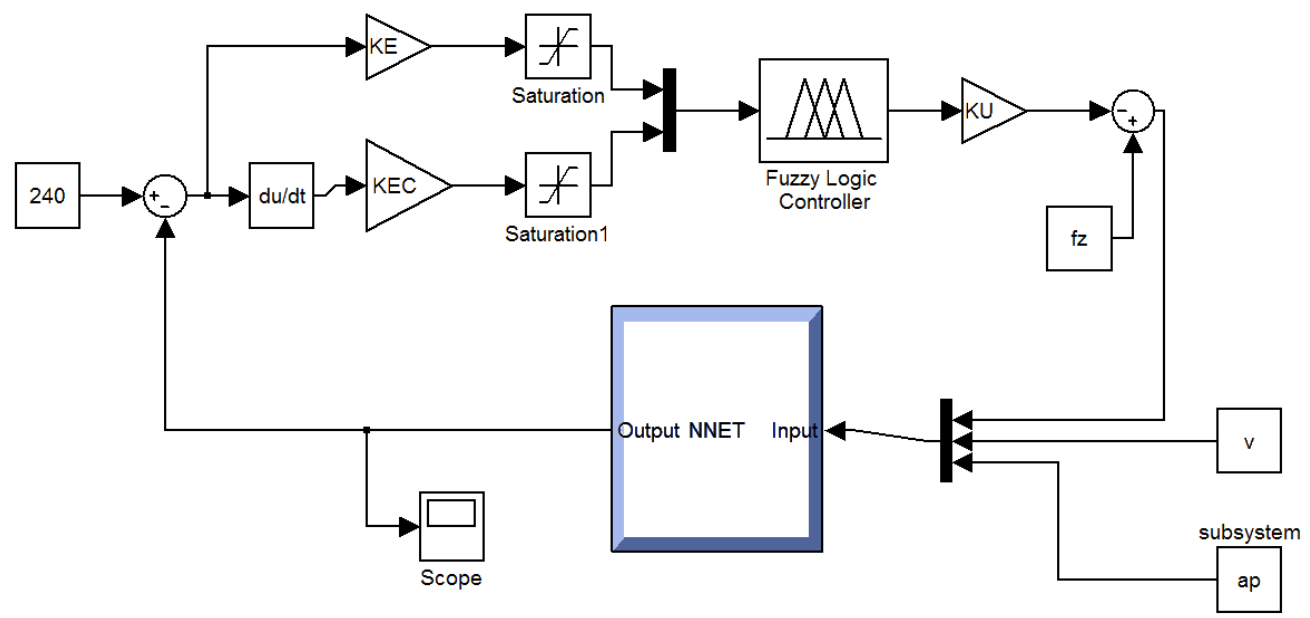

Figure 4. Simulation block diagram of fuzzy constant force control in Matlab/Simulink

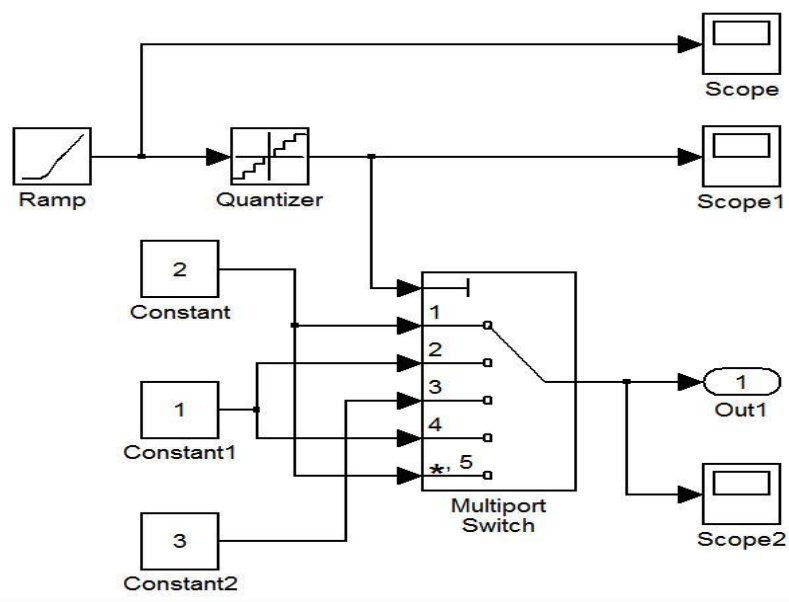

Figure 5. The subsystem of cutting depth step change

High speed milling constant force control of a Matlab/Simulink simulation model diagram is shown in Figure 4. The Fuzzy Logic Toolbox in Simulink doesn't need to do a complicated fuzzification, fuzzy inference and defuzzification, only need to set the corresponding parameter, can quickly get fuzzy controller. But the fuzzy control rules are fixed, therefore, establishing $\mathrm{S}$ functions in Matlab according to formula (3) and (4), in order to meet the online self-tuning fuzzy rules. A Subsystem module is established to achieve the change rule of cutting depth, as shown in Figure 5. Oblique wave output Ramp is quantitatively outputted by the module Quantizer, the three cutting depth corresponding value is $2 \mathrm{~mm}, 1 \mathrm{~mm}$ and 3 $\mathrm{mm}$. When Multiport Switch port value is 1 , the value of Constant is outputted from the first input port to the output port. The rest is similar. Setting the Multiport Switch time, can be realized input different cutting depth at different times.

The theory of fuzzy control constant force simulation is as follows: given initial per tooth 
feed $f_{z}$ and the axial cutting depth $a_{p}$, cutting speed $v$, simulated in a high speed milling model, the actual cutting force $F$ can be computed. Compared with a given reference force $F_{r e f}$, the difference and the change rate of difference can be inputted fuzzy controller. The output control variable $\Delta f_{z}$ through fuzzy control rules is feedbacked to the CNC system. Summed with the initial per tooth feed, the actual per tooth feed is computed. The value is input to the process model, and adjusts repeatedly, until the output of the milling force is close to reference milling force.

Specific simulation condition is: milling cutter diameter $12 \mathrm{~mm}$, double edge; spindle speed $n=12000 \mathrm{r} / \mathrm{min}$, reference forces $F_{r e f}=240 \mathrm{~N}$, per tooth feed $f_{z}=0.02 \mathrm{~mm} / \mathrm{r}, K_{E}=0.8, K_{E C}=0.25, K_{U}=2.3$. When the axial cutting depth $a_{p}$ changing, the change rules of cutting force $F$, the material removal rate MRR is researched. The curve of axial cutting depth changing with time is shown in Figure 6.

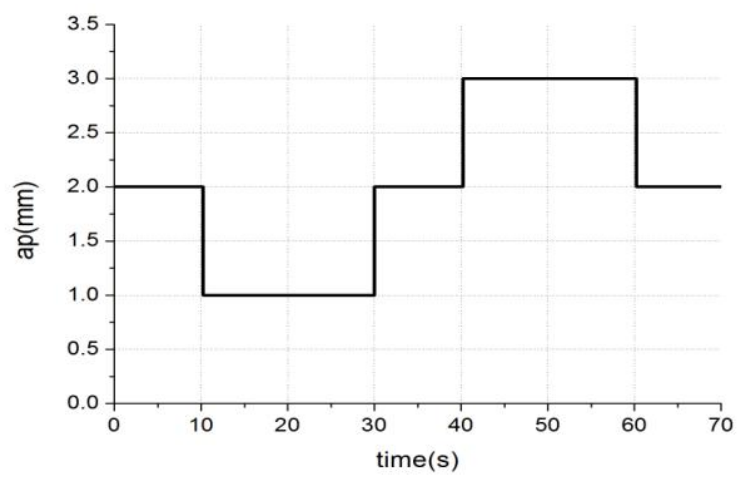

Figure 6. Variation rule of axial cutting depth with time

The simulation results not applying fuzzy control are shown in Figure 7(a) and using fuzzy control simulation results are shown in Figure 7(b). From the graph, we can see: material removal rate is more stable by using fuzzy control, no obvious changes, and the average MRR is $18 \%$ higher than not using the fuzzy control. The cutting force can also quickly achieve and maintain near the reference cutting force $240 \mathrm{~N}$. The metal removal rate can be improved, and constant cutting force can be maintained by adjusting the cutting feed in a fuzzy control system, the processing efficiency enhancing obviously.
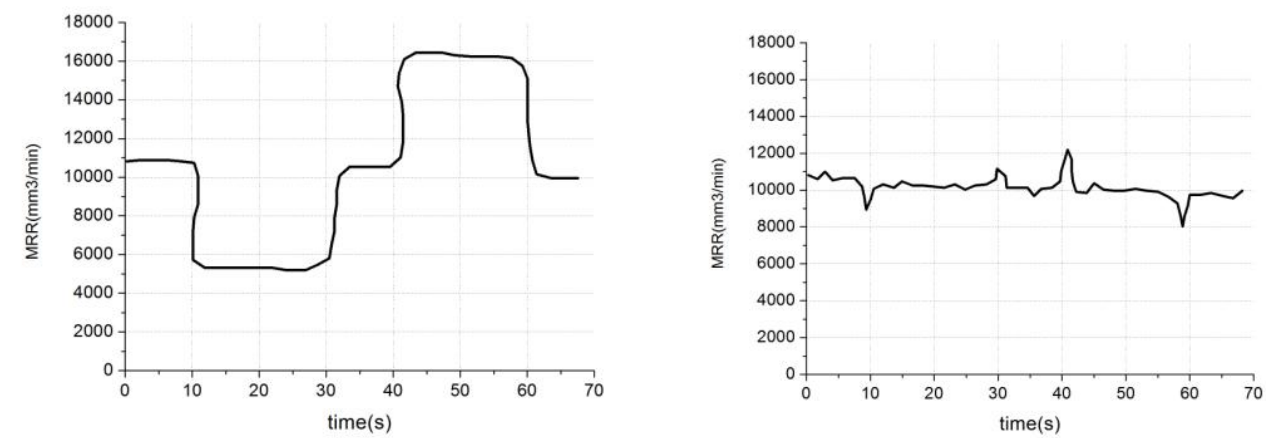


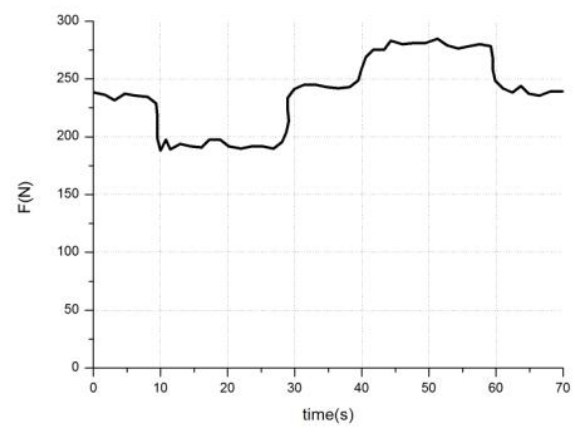

(a)

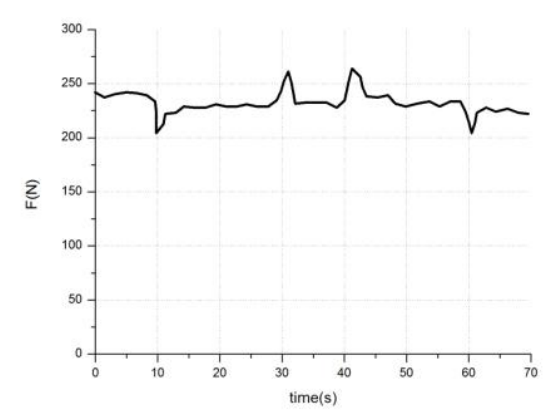

(b)

Figure 7. Simulation result (a) without fuzzy control (b)with fuzzy control

\section{Conclusions}

An adaptive control system is built which controlling the cutting force and maintaining constant roughness of the surface being milled by digital adaptation of cutting parameters. The experimental results show that not only does the milling system with the design controller have high robustness, and global stability but also the machining efficiency of the milling system with the adaptive controller is much higher than for traditional CNC milling system. Experiments have confirmed efficiency of the adaptive control system, which is reflected in improved surface quality and decreased tool wear.

(1)A fuzzy controller is designed suitable for high speed milling. The fuzzy rules are online self-tuning, and output scaling factor is online adaptive, both can well meet the requirements of constant force cutting. The controller algorithm is simple and can be used for real-time online control, has strong adaptability and good robust performance.

(2)The high speed milling process is modeled by using BP neural network, overcoming the shortage of milling process modeled by the traditional transfer function, which is more accurate to establish the relationship between cutting force and cutting parameters.

The results of experiments demonstrate the ability of the proposed system to effectively regulate peak cutting forces for cutting conditions commonly encountered in high speed milling operations. The high accuracy of results within a wide range of machining parameters indicates that the system can be practically applied in industry.

\section{Acknowledgements}

The authors gratefully acknowledge the technical and financial support of the science and technology major projects "High-end CNC machine tools and basic manufacturing equipment" (number: 2010 zx04014-051) granted by the Ministry of Science and Technology.

\section{References}

[1] J. Balic, "A new NC machine tool controller for step-by-step milling”, Int. J. Adv. Manuf. Technol, vol. 18, (2001), pp. 399-403.

[2] Y. Liu, L. Zuo and C. Wang, "Intelligent adaptive control in milling process", International Journal of Computer Integrated Manufacturing, vol. 12, (1999), pp. 453-460.

[3] L. A. DobrzaĚski, K. Golombek, J. Kopac and M. Sokovic, "Effect of depositing the hard surface coatings on properties of the selected cemented carbides and tool cermets", J. Mater, Process, Technol, (2004), pp. 157-158, pp. 304-311. 
[4] X. -H. Yao, Y. -H. Peng and T. -J. Chen, "Integrated adaptive fuzzy control of machining process", China Mechanical Engineering, vol. 9, no. 10, (1998), pp. 55-56.

[5] C. Tao, Z. Li and Y. -M. Liu, "Study on intelligent adaptive control in CNC cutting process", China Mechanical Engineering, vol. 10, no. 1, (1999), pp. 26-31.

[6] X. -F. Yao, X. -Q Yao and L. Can, "Control Evolution and Case Study for Uncertain Machining Process", Journal of basic science and engineering, vol. 18, no. 1, (2010), pp. 177-186.

[7] X. -Y. Lai, B. -Y Ye and C. -Y Yan, "Adaptive fuzzy control of milling process based on fieldbus", Journal of South China University of Technology(Natural Science Edition), vol. 33, no. 5, (2005), pp. 7-10.

[8] M. Liang, T. Yeap and A. Hermansyah, "Fuzzy control of spindle torque for industrial CNC machining", International Journal of Machine Tools and Manufacture, vol. 43, (2003), pp. 1497-1508.

[9] J. -H. Zhao, "On-line self-truning fuzzy control in the machining process", Journal of Guangzhou University(Natural Science Edition), vol. 3, no. 3, (2004), pp. 235-238.

[10] B. -P. Ma and Z. -G. Xu, "The fuzzy controller with on-line self adjustable proportional factor", Process Automation Instrumentation, no. 8, (2000), pp. 20-22.

[11] T. J. Procky and E. H. Mamdni, "A Limistic Self-Organizing Process Controller”, Automatica, vol. 15, (1979), pp. 15-30.

[12] Y. S. Tarng and Y. S. Wang, “An Adaptive Fuzzy Control system for Turning Operations”, Int. J. Mach. Tools Manufact, vol. 33, no. 6, (1993), pp. 761-771.

[13] Q. -S Pang, S. -G Xiao and M. -M. Song, "Model reference adaptive control of numerical control machine tool machining process", Machine Tool \& Hydraulics, vol. 37, no. 03, (2009), pp. 53- 55.

[14] M. -Q. Yu and X. -F Yao, "Fuzzy adaptive PID control of a machining process system", Control Engineering of China, vol. 16, no. S2, (2009), pp. 42-44.

[15] H. -B. Sun and S. -Q. Li, "General nonlinear system MRAC based on neural network", Application Research of Computers, (2009), vol. 26, no. 11, pp. 4169- 4171.

[16] T. Huang, Z. -Y. Yang and D. -W. Zhang, "Application of fuzzy control with self-tuning factor to NC milling process", Journal of Vibration Engineering, vol. 13, no. 1, (2000), pp. 53-60.

[17] X. -L Jiang, S. -M. Cheng and Y. -S. Li, "Algorithm for model reference adaptive control based on neural network", Journal of Huazhong University of Science and Technology(Natural Science Edition), vol. 31, no. 01, (2003), pp. 4-6.

[18] C. -Y. Yan, X. -X. Deng and W. -G. Li, "Adaptive control of machining process based on neural network", Acta Agriculturae Universitatis Jiangxiensis, vol. 27, no. 6, (2005), pp. 916-919.

\section{Authors}

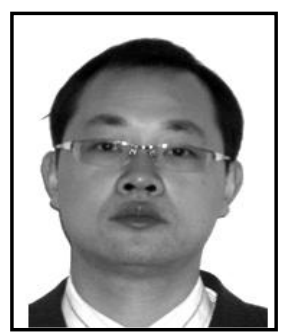

\section{Yunlin Huang}

He received his M.Eng. in Mechanical Engineering (2006) from Anhui University of technology, now he is studying doctoral degree in Mechanical Engineering in Nanjing University of Science and Technology. His current research interests include cutting stability and high speed cutting.

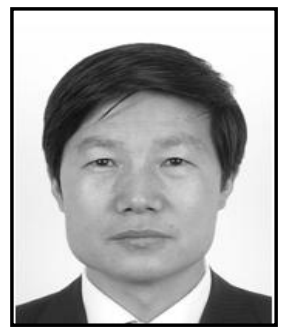

\section{Juntang Yuan}

He received his M.Eng. in Mechanical Engineering (1988) and $\mathrm{PhD}$ in Mechanical Engineering (2002) from Nanjing University of Science and Technology. Now he is full professor of Mechanical engineering at Mechanical engineering Department, Nanjing University of Science and Technology. His current research interests include advanced manufacturing technology and equipment. 
International Journal of Control and Automation Vol.7, No.5 (2014) 